\title{
Rate Expression of Fischer-Tropsch Synthesis Over Co-Mn Nanocatalyst by Response Surface Methodology (RSM)
}

\author{
Mohsen Mansouri, Hossein Atashi*, Mir Mohammad Khalilipour, Naimeh Setareshenas, and Farhad Shahraki \\ Department of Chemical Engineering, University of Sistan \& Baluchestan, Zahedan 98164-161, Iran. \\ ${ }^{*}$ E-mail: atashi.usb@gmail.com
}

(Received July 14, 2013; Accepted November 10, 2013)

\begin{abstract}
The effect of operating conditions (temperature and the partial pressures of $\mathrm{H}_{2}$ and $\mathrm{CO}$ ) on the reaction rate of Fischer-Tropsch synthesis (FTS) were investigated by carrying out experiments according to a Box-Behnken design (BBD), and were mathematically modeled by using response surface methodology (RSM). The catalyst used was a nano-structured cobalt/manganese oxide catalyst, which was prepared by thermal decomposition. The rate of synthesis was measured in a fixed-bed micro reactor with $\mathrm{H}_{2} / \mathrm{CO}$ molar feed ratio of $0.32-3.11$ and reactor pressure in the range of 3-9.33 bar at space velocity of $3600 \mathrm{~h}^{-1}$ and a temperature range of $463.15-503.15 \mathrm{~K}$, under differential conditions (CO conversion below $2 \%$ ). The results indicated that in the present experimental setup, the temperature and the partial pressure of CO were the most significant variables affecting reaction rate. Based on statistical analysis the quadratic model of reaction rate of FTS was highly significant as p-value 0.0002 .
\end{abstract}

Key words: Fischer-Tropsch synthesis, RSM, Rate of CO consumption, Cobalt-manganese nanocatalyst

\section{INTRODUCTION}

The Fischer-Tropsch synthesis (FTS) is an interesting and promising pathway for the conversion of natural gas to transportation fuels. ${ }^{1}$ While process variables such as temperature, pressure, gas flow rate and ratio in the $\mathrm{H}_{2} / \mathrm{CO}$ feed are very important, the major determinants of reaction rate and product distribution are the catalyst used. Among the candidate transient metals only $\mathrm{Co}$ and $\mathrm{Fe}$ based catalysts due to their cost, activity and selectivity have been developed for industrial uses. ${ }^{2}$ It is shown that the addition of $\mathrm{Mn}$ to Fe or Co catalysts leads to a significant increase in high olefin formation and a decrease in methane activity. ${ }^{3,4}$ Mixed metal catalysts are often capable of activity, selectivity, or have a stability, that is unobtainable with a single component and it is believed that bimetallic catalysts system due to the thermodynamic and kinetic limitations of the reaction are more able to raise the value of the light olefins. ${ }^{1,5}$

The rate description of the FT reaction is very important task for the industrial practice, being a prerequisite for the industrial process design, optimization and simulation. Rate of CO conversion values in the FTS have been achieved in various temperatures, pressures, catalyst weights and $\mathrm{H}_{2} / \mathrm{CO}$ molar ratios. If the used experimental data is enough, a reaction rate study will be reliable; in the rate of $\mathrm{CO}$ consumption study, the equation's accuracy of chem- ical reactions increases as the number of experimental data increases. Provision of the numerous data using the fixed bed micro-reactor is a time-consuming and costly procedure. Thus a mathematical model that can estimate the major experimental data will be very valuable and interesting in industry.

In recent years, design of experiments (DOE) and statistical tools has been attracted researchers and several experimental studies have been conducted through DOE. ${ }^{6-11}$ DOE is a structured and systematized method of experimentation in which all factors are varied simultaneously over a set of experimental runs in order to determine the relationship between factors (i.e., interaction effects between experimental condition parameters) affecting the output response of the FTS process.

Having a variety of statistical tools, the response surface methodology (RSM) is an efficient procedure in scientific studies. This methodology is a collection of statistical techniques for designing of experiments, building the models, evaluating the effects of factors and searching for the optimum conditions. The purpose of RSM is establishing the systematic modeling, demonstrating and optimizing the behaviors via regression/statistical/graphical tools which lead to more efficient understanding of the complicated processes. In the last few years, RSM has been applied to optimize and evaluate interactive effects of independent factors in numerous chemical and biochemical processes 
such as, wastewater treatment, ${ }^{12} \mathrm{CO}_{2}$ adsorption, ${ }^{13}$ steam reforming of methanol, ${ }^{14}$ thermoacoustic refrigerator, ${ }^{15}$ trireforming of methane. ${ }^{16}$ Although statistical methodologies and RSM have been used extensively in various scientific fields as well as catalysis science, ${ }^{6-9}$ a few studies for $\mathrm{CO}$ hydrogenation are based on statistical methodologies. ${ }^{10,11}$ However, the rate of $\mathrm{CO}$ consumption and the effect of interaction of operating conditions using response surface methodology based on the Box-Behnken design (BBD) to develop model for reaction rate of FTS have not been reported.

The objective of this study is to assess the rate of the $\mathrm{CO}$ hydrogenation on nano-structured $\mathrm{Co}-\mathrm{Mn}$ catalyst which was prepared by thermal decomposition of $\left[\mathrm{Co}\left(\mathrm{NH}_{3}\right)_{4} \mathrm{CO}_{3}\right] \mathrm{MnO}_{4}$ precursor. The individual and mutual effects of the experimental variables, temperature and the partial pressures of $\mathrm{H}_{2}$ and $\mathrm{CO}$, on the reaction rate in the FTS were investigated using the statistical design of experiments and RSM. Empirical mathematical models for the rate expression of FTS as function of the most significant rate expression of FTS variables have been developed and validated based on regression techniques.

\section{MATERIALS AND METHODS}

\section{Catalyst Synthesis and Characterization ${ }^{17}$}

The catalyst used in the present study was prepared using a thermal decomposition precursor as follows. An aqueous solution $(60 \mathrm{ml})$ of ammonium carbonate $(20 \mathrm{~g})$ was added to a concentrated aqueous $\mathrm{NH}_{3}(60 \mathrm{ml})$, and the mixture was stirred at room temperature for $30 \mathrm{~min}$. To this solution was then added an aqueous solution $(30 \mathrm{ml})$ of $\mathrm{Co}\left(\mathrm{NH}_{3}\right)_{2} \cdot 6 \mathrm{H}_{2} \mathrm{O}(15 \mathrm{~g})$ and the resulting solution was stirred at room temperature for $1 \mathrm{~h}$. Then, $8 \mathrm{ml}$ of $30 \%$ hydro- gen peroxide was dropwise added into the solution, stirring continuously. The final solution was filtered and left for slow evaporation in air until water-soluble $\left[\mathrm{Co}\left(\mathrm{NH}_{3}\right)_{4} \mathrm{CO}_{3}\right] \mathrm{NO}_{3}$ crystals were obtained. $\mathrm{KMnO}_{4}(1 \mathrm{mmol})$ was added to a solution containing $\left[\mathrm{Co}\left(\mathrm{NH}_{3}\right)_{4} \mathrm{CO}_{3}\right] \mathrm{NO}_{3}(1 \mathrm{mmol})$ in $100 \mathrm{ml}$ water and the mixture was stirred for several minutes. The precipitates $\left(\left[\mathrm{Co}\left(\mathrm{NH}_{3}\right)_{4} \mathrm{CO}_{3}\right] \mathrm{MnO}_{4}\right.$ precursor) were recovered by centrifugation and washed by distilled water and then dried in ambient air at $30^{\circ} \mathrm{C}$ overnight. In order to obtain the final nano-catalyst, the precursor was calcined at $400{ }^{\circ} \mathrm{C}$ in static air in the electric furnace for $4 \mathrm{~h}$. The gray powder, nano-structured Co-Mn oxide catalyst was formed and kept in desiccator.

Physical properties and SEM images of precursor and calcined catalysts (before and after the reaction) are shown in Table 1 and Fig. 1, respectively. The SEM observations have shown differences in morphology of precursor and calcined catalysts (before and after the reaction) and indicate that these materials are made of nano-metric particles.

\section{Catalyst Testing}

A schematic representation of the experimental setup is shown in Fig. 2. Fischer-Tropsch synthesis was carried out in a fixed-bed micro-reactor made of stainless steel with an inner diameter of $20 \mathrm{~mm}$. All gas lines to the reactor bed were made from $1 / 4$ stainless steel tubing. Three mass flow controllers (Brooks, Model 5850E) equipped with a four-channel read out and control equipment (Brooks 0154) were used to adjust automatically the flow rate of the inlet gases $\left(\mathrm{CO}, \mathrm{H}_{2}\right.$, and $\mathrm{N}_{2}$ with purity of $\left.99.99 \%\right)$. The mixed gases in the mixing chamber passed into the reactor tube, which was placed inside a tubular furnace (Atbin, Model ATU 150-15) capable of producing a temperature up to $1500{ }^{\circ} \mathrm{C}$ and controlled by a digital programmable

Table 1. Physical properties of the precursor and nanocatalyst

\begin{tabular}{lccc}
\hline Sample & BET surface area $\left(\mathrm{m}^{2} / \mathrm{g}\right)$ & Pore volume $\left(\mathrm{cm}^{3} / \mathrm{g}\right)$ & Average pore diameter $(\mathrm{nm})$ \\
\hline$\left[\mathrm{Co}\left(\mathrm{NH}_{3}\right)_{4} \mathrm{CO}_{3}\right] \mathrm{MnO}_{4}$ & 11.32 & 0.12 & 16.89 \\
Nano catalyst & 74.28 & 0.21 & 13.32 \\
\hline
\end{tabular}
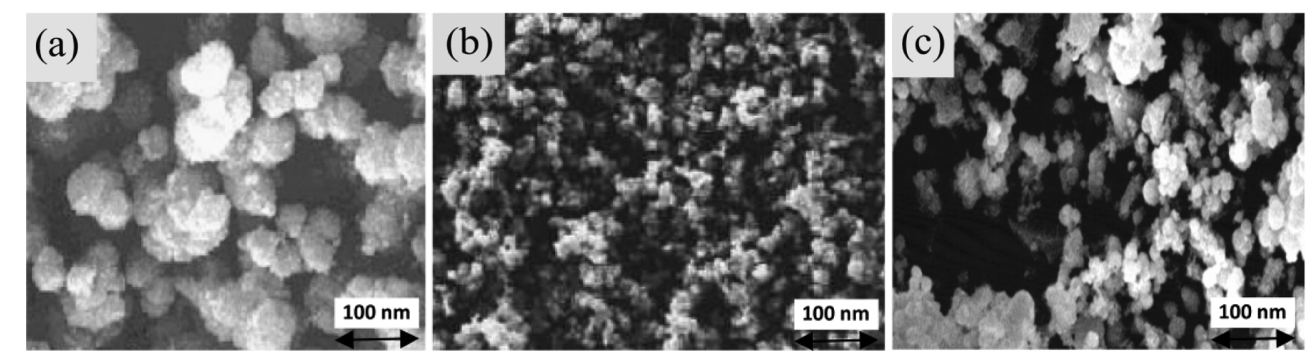

Figure 1. SEM micrographs of (a) precursor, (b) calcined catalyst before the test, and (c) calcined catalyst after the test. 


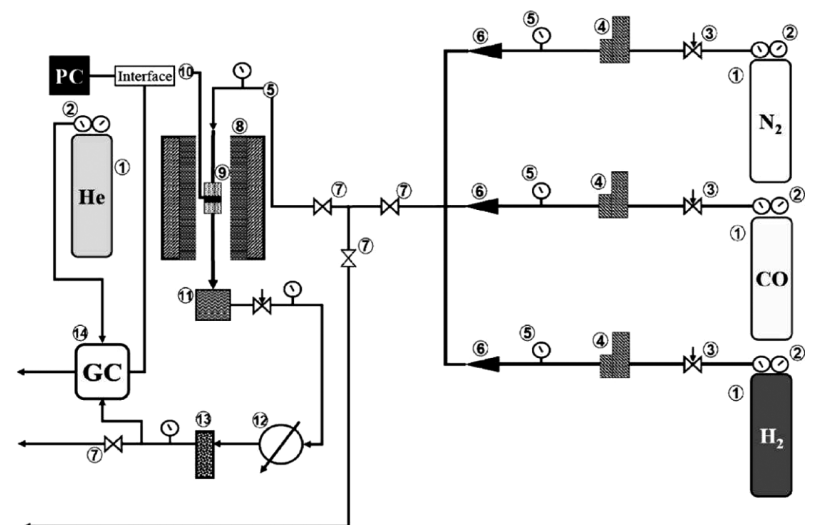

Figure 2. Schematic representation of the reactor used. (1) Gas cylinders, (2) pressure regulators, (3) needle valves, (4) mass flow controllers, (5) monometers, (6) non-return valves, (7) ball valves, (8) tubular furnace, (9) reactor, (10) catalyst bed, (11) trap, (12) condenser, (13) silica gel column, and (14) gas chromatograph (GC).

controller (DPC). The reactor tube was constructed from stainless steel tubing; internal diameter of $9 \mathrm{~mm}$, with the catalyst bed situated in the middle of the reactor. The reaction temperature was controlled by a thermocouple inserted into catalyst bed and visually monitored by a computer equipped with software. Some thermocouples inserted in the catalyst bed for monitoring the inlet, outlet and bed temperatures by a DPC. The meshed catalyst $(1.0 \mathrm{~g})$ was held in the middle of the reactor using quartz wool. It consist of an electronic back pressure regulator which can control the total pressure of the desired process using a remote control via the TESCOM software package integration that improves or modifies its efficiency that is capable for working on pressure ranging from atmospheric pressure to $34 \mathrm{bar}$. The catalyst was pre-reduced in situ atmospheric pressure in a flowing $\mathrm{H}_{2}-\mathrm{N}_{2}$ stream $\left(\mathrm{N}_{2} / \mathrm{H}_{2}=1\right.$, flow rate of each gas = $30 \mathrm{ml} / \mathrm{min}$ ) at $300^{\circ} \mathrm{C}$ for $12 \mathrm{~h}$ before synthesis gas exposure.

The catalysts were extremely fine particles so intraparticle diffusion could be neglected. The gas hourly space velocity (GHSV) increased to the value in which the CO conversion was almost the same for a variety of catalyst weight which indicates that external diffusion can be neglected above this GHSV. Hence, the experiments for the rate expression were conducted free from internal and external mass transfer limitations. Experiments were carried out with mixtures of $\mathrm{H}_{2}, \mathrm{CO}$ and $\mathrm{N}_{2}$ in a temperature range of 463.15-503.15 K, $\mathrm{H}_{2} / \mathrm{CO}$ molar feed ratio of 0.32/1-3.11/1, pressure range of 3-9.33 bar and GHSV $=3600 \mathrm{~h}^{-1}$ after $10-15 \mathrm{~h}$ of reaction to obtain a steady-state activity. Reactant and product streams were analyzed on-line using a gas chromatograph (Varian, Model 3400 Series) equipped with a 10-port sampling valve (Supelco company, USA, Visi Model), a sample loop, flam ionization detector (FID) and thermal conductivity detector(TCD). The contents of the sample loop were injected automatically into a packed column (Hayesep DB, Altech Company, USA, 1/8 OD, $10 \mathrm{~m}$ long, and particle mesh 100/120). Helium was employed as a carrier gas for optimum sensitivity (flow rate $=30 \mathrm{ml} /$ $\mathrm{min}$ ). The calibration was carried out using various calibration mixtures and pure compounds obtained from American Matheson Gas Company (USA). GC control and collection of all chromatograms were performed via an IF-2000 Single channel data interface (TG Co, Tehran, Iran) using Windows ${ }^{\circledR}$ environment. Typically, the experimental reaction rate was determined as follows:

$$
-r_{C O}=\frac{N_{\mathrm{CO}, \text { in }}-N_{\mathrm{CO}, \text { out }}}{W}
$$

and

$$
N_{\mathrm{CO}, \text { in }}=\frac{v_{i n} P_{C O}^{0}}{R T}
$$

where, $r_{\mathrm{CO}}$ is $\mathrm{CO}$ consumption rate ( $\left.\mathrm{mol} \mathrm{CO} g_{c a t}{ }^{-1} \mathrm{~min}^{-1}\right)$, $N_{\mathrm{CO}, \text { in }}$ and $N_{\mathrm{CO}, \text { out }}$ are $\mathrm{CO}$ molar flow rates of feed and effluent stream $\left(\mathrm{mol} \mathrm{min}^{-1}\right)$, respectively. $W$ is catalyst mass $(\mathrm{g}), v_{\text {in }}$ represents the volumetric flow rate of feed gas $\left(\mathrm{cm}^{3} \mathrm{~min}^{-1}\right), P_{\mathrm{CO}}^{0}$ is inlet $\mathrm{CO}$ partial pressure (bar), $T$ is temperature $(\mathrm{K})$ and $R$ is the general gas constant $\left(\mathrm{cm}^{3}\right.$ bar $\left.\mathrm{mol}^{-1} \mathrm{~K}^{-1}\right)$.

\section{Experimental Design and Response Surface Regression}

RSM is a collection of mathematical and statistical techniques for empirical model building. By careful design of experiments, the objective is to develop quadratic model for independent parameters and to test response surface model by analysis of variance (ANOVA). In this method the response and independent variables are defined by input and output variable respectively. An experiment is a series of tests, called runs, in which changes are made in the input variables in order to identify the reasons for changes in the output response. By cognizance of significant factors from previous works or carrying out factorial design, the mathematical model could be developed by using more complex experimental designs such as central composite designs (CCD), the BBD, Doehlert matrix and three-level full factorial design. ${ }^{18}$ Ferreira et al.$^{19}$ showed that the BBD method where the factor number is higher than 2 is much more efficient in comparison to other response surface designs for the quadratic model. The most important feature of $\mathrm{BBD}$ is avoiding of suggesting runs which all factors are 
simultaneously at their highest or lowest levels. For a problem involving $k$ factor (independent variable) $\mathrm{BBD}$ date point consist of $2 k(k 1)+C_{0}$ points (where $k$ is number of factors and $C_{0}$ is the number of central points). By linear regression the $\mathrm{BBD}$ method use the experimental result to determine the coefficients of the second-degree polynomial equations.

Analysis of variance (ANOVA) was used for graphical analyses of the data to obtain the interaction between the process variables and the responses. The quality of the fit polynomial model was expressed by the coefficient of determination $\mathrm{R}^{2}$, and its statistical significance was checked by the Fisher's F-test in the same program. Model terms were evaluated by the P-value (probability) with $95 \%$ confidence level. Three-dimensional plots and their respective contour plots were obtained for reaction rate of FTS based on effects of the three variables (temperature, partial pressure of $\mathrm{CO}$ and partial pressure of $\mathrm{H}_{2}$ ) at three levels.

As shown in Fig. 3, this rotatable experimental plan was carried out as a BBD consisting of 15 experiments for the rate expression of FTS. The factors (variables) in this experiment were the temperature $\left(\mathrm{X}_{1}\right)$, partial pressure of $\mathrm{CO}$ $\left(\mathrm{X}_{2}\right)$ and partial pressure of $\mathrm{H}_{2}\left(\mathrm{X}_{3}\right)$. These three variables are taken into consideration according to previous study as significant factor in FTS. ${ }^{1-5,17-23}$ The operating region

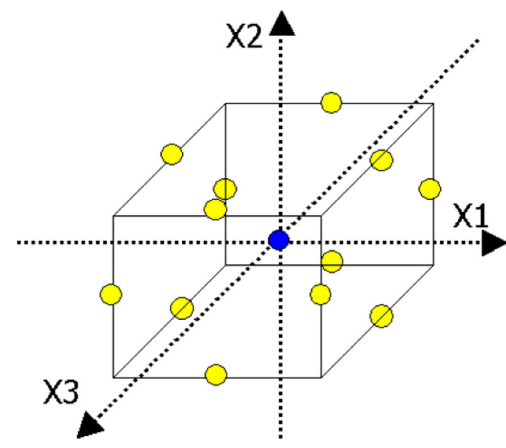

Figure 3. Schematic diagram of Box Behnken design (BBD) as a function of $\mathrm{X}_{1}$ (temperature), $\mathrm{X}_{2}$ (partial pressure of $\mathrm{CO}$ ), and $\mathrm{X}_{3}$ (partial pressure of $\mathrm{H}_{2}$ ).

and the levels of the variables in actual and coded values are shown in Table 2 variables use for FTS. The response surface model (RS-model) with interaction terms was developed for the reaction rate of FTS using Eq. (3).

$$
\hat{y}=b_{0}+\sum_{i=1}^{k} b_{i} X_{i}+\sum_{i=1}^{k} b_{i i} X_{i}^{2}+\sum_{i<j}^{k} b_{i j} X_{i} X_{j}
$$

where $\hat{y}$ is the predicted response, $X_{i}$ is the coded variables, and $b_{0}, b_{i}, b_{i i}, b_{i j}$ are the regression coefficients (i.e. offset term, main, quadratic and interaction terms) of BBD method.

Table 2. Designed variables and their coded and actual values used for experimental design.

\begin{tabular}{|c|c|c|c|c|c|}
\hline Design Variables (factors) & Coded variables & Actual variables & \multicolumn{3}{|c|}{ Actual values of coded levels } \\
\hline Temperature $(\mathrm{K})$ & $\mathrm{X}_{1}$ & $T$ & 463.15 & 483.15 & 503.15 \\
\hline Partial pressure of $\mathrm{CO}$ (bar) & $\mathrm{X}_{2}$ & $P_{C O}$ & 0.9 & 1.85 & 2.8 \\
\hline Partial pressure of $\mathrm{H}_{2}$ (bar) & $\mathrm{X}_{3}$ & $P_{\mathrm{H}_{2}}$ & 0.9 & 1.85 & 2.8 \\
\hline
\end{tabular}

Table 3. Experimental values for reaction rate of FTS after $10-15 \mathrm{~h}$ of starting reaction at given conditions in a fixed bed reactor

\begin{tabular}{cccccc}
\hline \multirow{2}{*}{ No } & \multicolumn{3}{c}{ Factors (input variables) } & \multirow{2}{*}{ CO conversion $(\%)$} & \multirow{2}{*}{ Response $^{\circledR} \times 10^{3}\left(\mathrm{~mol}_{\mathrm{cat}^{-1}} \mathrm{~min}^{-1}\right)$} \\
\cline { 2 - 4 } & $T(\mathrm{~K})$ & $P_{C O}$ (bar) & $P_{H_{2}}$ (bar) & 0.9 & 12.632 \\
2 & 463.15 & 0.9 & 1.85 & 1.42 & 18.329 \\
3 & 503.15 & 0.9 & 1.85 & 0.73 & 31.879 \\
4 & 463.15 & 2.8 & 1.85 & 1.22 & 48.922 \\
5 & 503.15 & 2.8 & 1.85 & 0.77 & 22.106 \\
6 & 463.15 & 1.85 & 0.9 & 1.13 & 30.137 \\
7 & 503.15 & 1.85 & 0.9 & 0.88 & 25.319 \\
8 & 463.15 & 1.85 & 2.8 & 1.29 & 34.397 \\
9 & 503.15 & 1.85 & 2.8 & 0.89 & 11.973 \\
10 & 483.15 & 0.9 & 0.9 & 0.95 & 39.943 \\
11 & 483.15 & 2.8 & 0.9 & 1.12 & 15.129 \\
12 & 483.15 & 0.9 & 2.8 & 1.09 & 45.987 \\
13 & 483.15 & 2.8 & 2.8 & 1.15 & 31.675 \\
14 & 483.15 & 1.85 & 1.85 & 1.09 & 30.126 \\
15 & 483.15 & 1.85 & 1.85 & 1.06 & 29.316 \\
\hline
\end{tabular}


Experimental data for reaction rate of FTS after $10-15 \mathrm{~h}$ of starting reaction in a fixed bed reactor over $\mathrm{Co}-\mathrm{Mn}$ nanocatalyst are given in Table 3 . The relationship between the three variables (temperature, partial pressure of $\mathrm{CO}$ and partial pressure of $\mathrm{H}_{2}$ ) and reaction rate of FTS was analyzed using response surface methodology (RSM).

\section{RESULT AND DISCUSSIONS}

\section{Analysis of Variance (ANOVA) and the Second-Order Model}

The ANOVA has been employed to check the significance of the second-order models. For response (reaction rate of FTS) the statistical significance of the second-order regression models was determined by F-value, which is measurement of variance of data about the mean, based on the ratio of mean square of group variance due to error. The analysis of variance of regression parameters of the response surface quadratic model for reaction rate of FTS is shown in Table 4. Based on ANOVA result the model is significant by the p-value of 0.0002 , similarly the model F-value of 56.39 implies that the model is also significant. The lack of fit term is non-significant ( 0.2385 i.e. greater than 0.05 ) which indicates that the quadratic model is valid and that the systematic variation does not exist in the hypothesized model. Moreover, the correlation coefficient $\left(\mathrm{R}^{2}=0.9902\right)$ obtained for FTS, indicating that good agreement between the calculated and observed results within the range of experiments. Therefore, the response surface model established in this study for predicting FTS was considered reasonable. The final model, in terms of coded and actual factors is expressed by the quadratic empirical equation in Table 5 .

The adequacy of the modeled approximation can be shown by diagnostic plots which are provided by RSM. Fig. 4 displays the normal probability of the residuals, to verify whether the standard deviations between the actual and the predicted response values follow a normal distribution. The results illustrated in Fig. 4 convey the general impression of a normal distribution of underlying errors, since the residuals fall near to a straight line; thus, there is no clear indication of non-normality of experimental results.

Actual value versus predicted value displays the real
Table 5. Quadratic model for FTS in terms of coded and actual factors
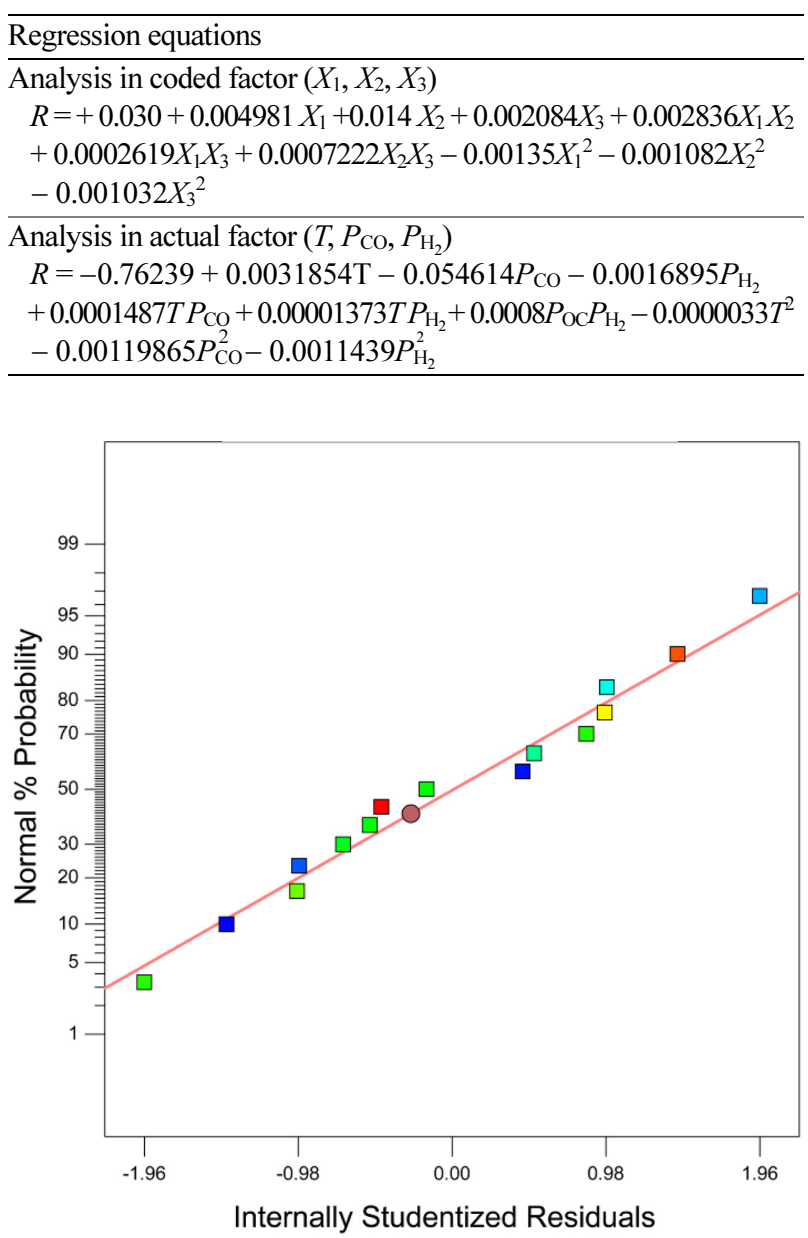

Figure 4. Normal probability plot of the studentized residuals for reaction rate of FTS.

responses data plotted against the predicted response is shown in Fig. 5. The predicted response values slightly deviated from the experimental data.

Fitting of the data to various models (linear, two factorial, quadratic and cubic) and their subsequent ANOVA showed that reaction rate of FTS was most suitably described with quadratic polynomial model. Using 10,5 , and $1 \%$ significance levels, a model is considered significant if the p-value (significance probability value) is less than $0.1,0.05$, and 0.001 , respectively. From the p-values presented in Table 6,

Table 4. Analysis of variance (ANOVA) for the FTS

\begin{tabular}{ccccccc}
\hline Source & $\mathrm{DF}^{\mathrm{a}}$ & $\mathrm{SS}^{\mathrm{b}}$ & $\mathrm{MS}^{\mathrm{c}}$ & $\mathrm{F}$-value & $\mathrm{R}^{2}$ & $\mathrm{R}^{2}{ }_{\text {adj }}$ \\
\hline Model & 9 & $1.757 \mathrm{E}-003$ & $1.952 \mathrm{E}-004$ & 56.39 & 0.9902 & 0.9727 \\
Residual & 5 & $1.731 \mathrm{E}-005$ & $3.462 \mathrm{E}-006$ & & & \\
\hline
\end{tabular}

${ }^{\mathrm{a} D F}$ : degree of freedom.

${ }^{\mathrm{b}} \mathrm{SS}$ : sum of squares.

${ }^{\mathrm{c}} \mathrm{MS}$ : mean square. 


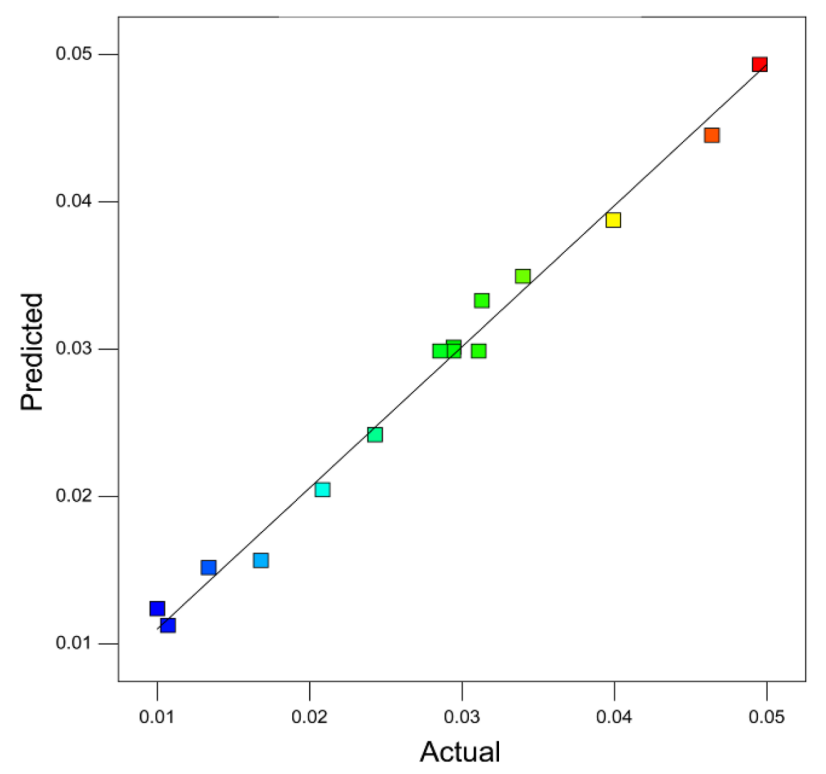

Figure 5. Predicted versus actual values plot for reaction rate of FTS.

Table 6. Analysis of variance for response

\begin{tabular}{lll}
\hline Source of variation & F-value & P-value \\
\hline Linear contribution & 96.6 & $0.0001^{* * *}$ \\
Cross-Product Contribution & 3.04 & $0.0930^{*}$ \\
Quadratic Contribution & 1.26 & 0.3835 \\
\hline *significant at $10 \%\left(\mathrm{p}\right.$-value); ${ }^{* *}$ Significant at $5 \%$ (P-value); and \\
***significant at $1 \%$ (p-value)
\end{tabular}

it can be concluded that for rate of $\mathrm{CO}$ consumption in FTS, linear contribution and cross-product contribution of the model were significant, whereas quadratic contribution of the model was insignificant. It is observed that there are tendencies in the linear regression fit, and the model explains the experimental range studied adequately.

In Table 7, associated P-values of the model are presented. The most significant factors of first-order (linear term) derive

Table 7. Associated p-values for response

\begin{tabular}{llll}
\hline Relationship & & Factor & P-value \\
\hline \multirow{4}{*}{ Main effects } & Linear & $T$ & $0.0006^{* * *}$ \\
& Linear & $P_{\mathrm{CO}}$ & $0.0001^{* * *}$ \\
& Linear & $P_{\mathrm{H}_{2}}$ & $0.0249^{* *}$ \\
\hline \multirow{4}{*}{ Interaction } & & $T \times P_{\mathrm{CO}}$ & $0.0285^{* *}$ \\
& \multirow{4}{*}{ Cross product } & $T \times P_{\mathrm{H}_{2}}$ & 0.7896 \\
& & $P_{\mathrm{CO}} \times P_{\mathrm{H}_{2}}$ & 0.4727 \\
& & $T$ & 0.222 \\
& \multirow{4}{*}{ Pure quadratic } & $P_{\mathrm{CO}}^{2}$ & 0.3147 \\
& & $P_{\mathrm{H}_{2}}$ & 0.3351 \\
\hline
\end{tabular}

*significant at $10 \%$ (p-value); ${ }^{* *}$ Significant at $5 \%$ (P-value); and $* * *$ significant at $1 \%$ (p-value) from the temperature and $P_{C O}$ with P-value of 0.0006 and 0.0001 , respectively. The last term of linear model has Pvalue of 0.0249 and is significant at $5 \%$. Also the first term of cross product $\left(T \times P_{C O}\right)$ is significant at $5 \%$ with $\mathrm{P}$-value of 0.0285 .

\section{Response Surface Estimation for Reaction Rate of FTS}

The response can be represented graphically, either in the three-dimensional space or as contour plots that help visualize the shape of the response surface. Canonical analysis using design expert $7^{\circledR}$ package is available to locate the stationary point of the response surface, and to decide whether it describes a maximum, minimum, or saddle point. Threedimensional (3D) and contour (2D) plots for the predicted responses were also formed, based on the model polynomial functions to assess the change of the response surface as shown in Figs. 6-8. The relationship between independent variables $\left(\mathrm{X}_{1}, \mathrm{X}_{2}, \mathrm{X}_{3}\right)$ can be also further understood by these plots. Since the model has more than two factors, one factor was held constant for each diagram; therefore, a total of three response surface diagrams were produced.

The syngas pressure is an important catalyst screening parameter. Variation of pressure is also applied in directing the FT process toward desired products. The results of FT catalyst screening at atmospheric and high pressure could yield different results. These differences could be interpreted in terms of different concentrations of reagents in gaseous and liquid phases, catalyst restructuring and deactivation. In commercial process, the FTS reaction usually operates under high pressure. An increase in total pressure would generally result in condensation of hydrocarbons, which are normally in the gaseous state at atmospheric pressure. Higher pressures and higher carbon monoxide conversions would probably lead to saturation of catalyst pores by liquid reaction products. ${ }^{20} \mathrm{~A}$ different composition of the liquid phase in catalyst pores at high synthesis gas pressures could affect the rate of carbon monoxide and hydrocarbon concentrations.

Temperature is a basic process variable that has a profound effect on the overall yield of a Fischer-Tropsch reactor. Temperature is normally used to control the distribution of products in the reaction, where one product may predominate at lower temperature and another predominates at higher temperature. The reaction temperature also has an impressive effect on the rate of $\mathrm{CO}$ conversion and catalytic performance of the catalysts. All reactions that take place in Fischer-Tropsch process are extremely exothermic, so temperature control is extremely necessary to ensure the reaction goes to selective products. 

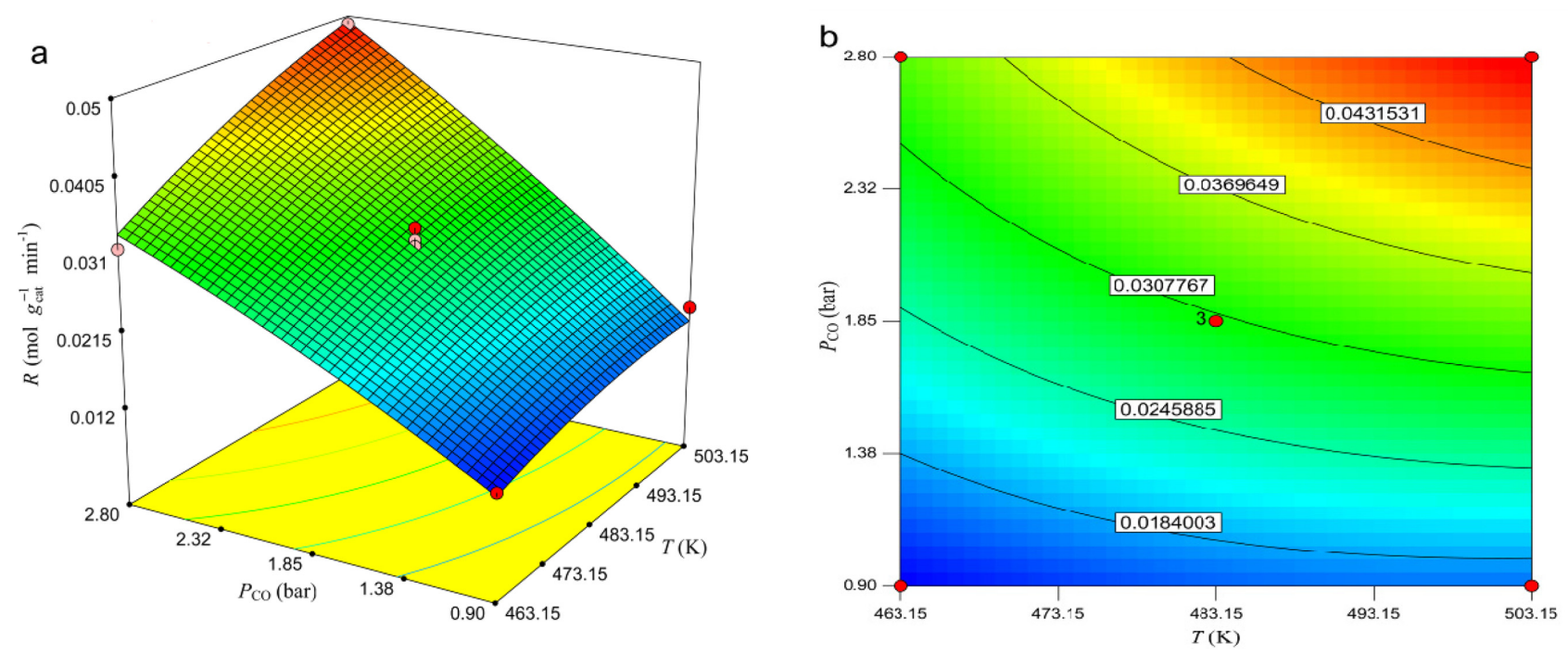

Figure 6. Response surface and contour plots of the reaction rate as a function of $T(\mathrm{~K})$ and $P_{\mathrm{CO}}$ (bar) for $P_{\mathrm{H}_{2}}=1.85$ bar.
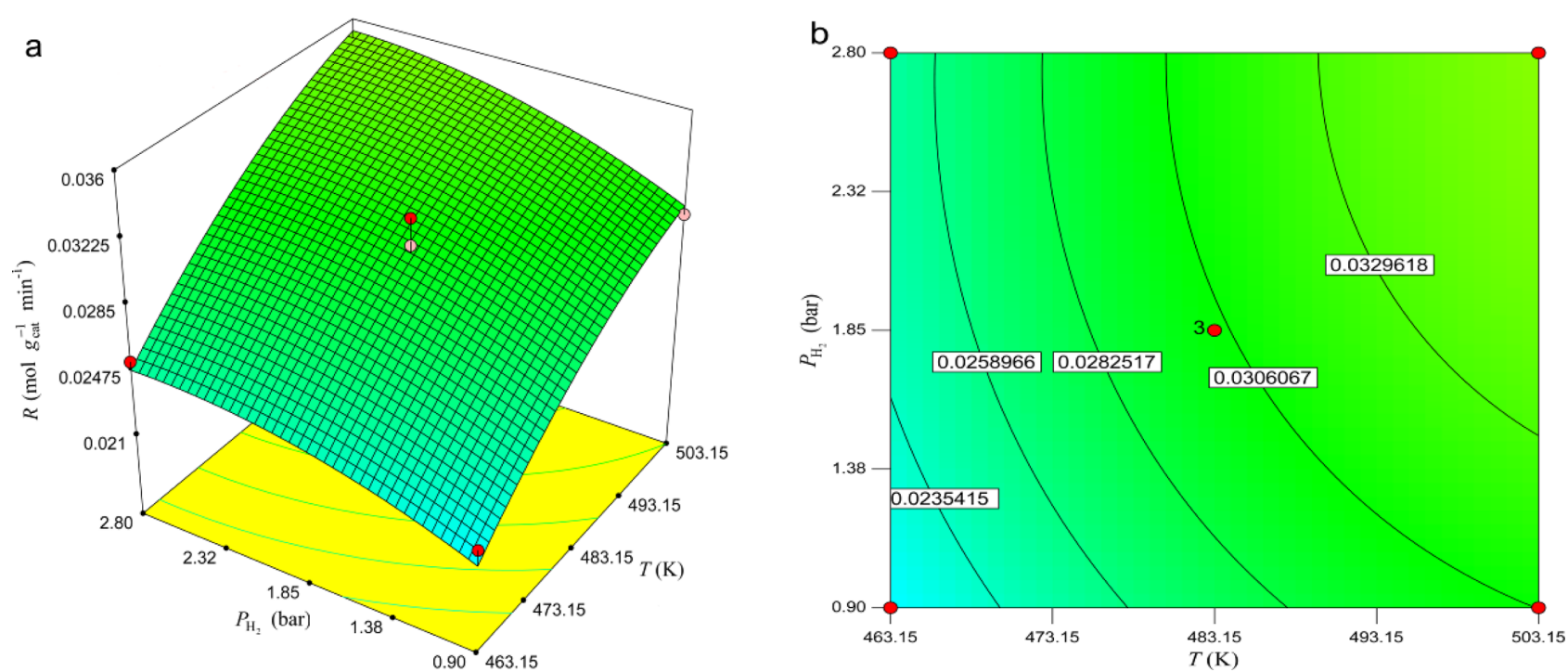

Figure 7. Response surface and contour plots of the reaction rate as a function of $T(\mathrm{~K})$ and $P_{\mathrm{H}_{2}}$ (bar) for $P_{\mathrm{CO}}=1.85$ bar.

As shown in Fig. 6, the effects of $P_{\mathrm{CO}}$ and temperature on the response was plotted where $P_{\mathrm{H}_{2}}$ was held constant at $1.85 \mathrm{bar}$. As can be seen, the interaction effect between the variables $P_{\mathrm{CO}}$ and temperature is obvious from both the 3-D response surface and the contour-line plots. The increase of $P_{\mathrm{CO}}$ leads to an increase of the reaction rate especially for higher temperature. For example, at the temperature of $503.15 \mathrm{~K}$, the increase of the $P_{\mathrm{CO}}$ leads to an enhancement

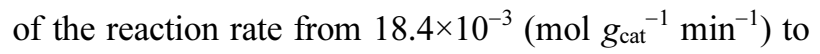
more than $43.15 \times 10^{-3}\left(\mathrm{~mol} \mathrm{~g}_{\mathrm{cat}}{ }^{-1} \mathrm{~min}^{-1}\right)$. For lower temperature $463.15 \mathrm{~K}$, increase in $P_{\mathrm{CO}}$ can only increase the consumption rate of $\mathrm{CO}$ from $18.4 \times 10^{-3}$ to $30.77 \times 10^{-3}$ mol $g_{\mathrm{cat}}{ }^{-1} \mathrm{~min}^{-1}$. Furthermore, the temperature effect on the reaction rate of FTS has been increased by increasing pressure of $\mathrm{CO}$ from 0.9 to 2.8 bar.

Fig. 7 shows the reaction rate as a function of temperature and $P_{\mathrm{H}_{2}}$ for fixed values of $P_{\mathrm{CO}}$ at $1.85 \mathrm{bar}$. The increase of temperature from $463.15 \mathrm{~K}$ to $503.15 \mathrm{~K}$ increase the rate of CO conversion, while the increase of $P_{\mathrm{H}_{2}}$ leads only to a slight increase of the reaction rate An increase in the reaction rate in the FTS with respect to the increase in the temperature and pressure has been cited in literature. ${ }^{21-27}$

In Fig. 8 the response surface and contour plots reveal the negligible interaction effects of the $P_{\mathrm{H}_{2}}$ and $P_{\mathrm{CO}}$ on the 

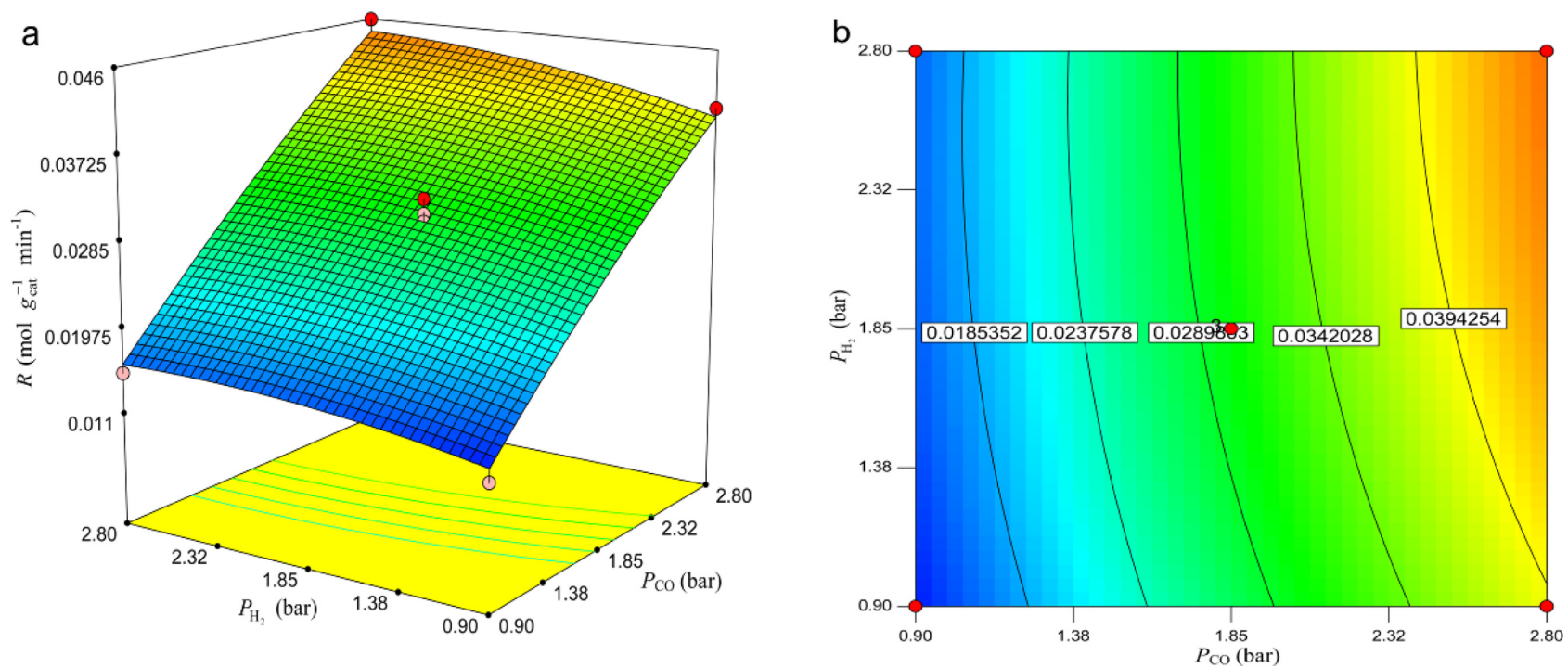

Figure 8. Response surface and contour plots of the reaction rate as a function of $P_{\mathrm{CO}}$ (bar) and $P_{\mathrm{H}_{2}}$ (bar) for $T=483.15 \mathrm{~K}$.

reaction rate. As it is expected, the response surface plot indicates that the rate of reaction increases with $P_{\mathrm{CO}}$ and within the tested range the effect of $P_{\mathrm{H}_{2}}$ on the rate of reaction is less than that of $P_{\mathrm{CO}}$. It should be pointed out that the interaction effect between $P_{\mathrm{CO}}$ and $P_{\mathrm{H}_{2}}$ is insignificant which is obtained earlier in Table 7.

Malek Abbaslou et al. ${ }^{28}$ reported that as the pressure increases, the supercritical media exhibits a liquid-like density, which can enhance extraction from the catalyst pores. This phenomenon helps $\mathrm{CO}$ and $\mathrm{H}_{2}$ adsorption onto active sites thereby increasing rate of $\mathrm{CO}$ conversion. It was also reported that the rate of different reactions of FTS that occur on the catalyst surface is strongly influenced by the reaction temperature. Similar to our results work, it has been reported that increasing the $\mathrm{CO}$ conversion is due to the enhanced concentration of active surface carbon species with increasing pressure and the improved collision probability of the catalysts and reactants. Thus the rate of reaction is enhanced. ${ }^{29}$

\section{CONCLUSIONS}

Design of experiments and response surface methodology (RSM) were applied to develop quadratic model for reaction rate of FTS applied for the conversion of syngas into a wide spectrum of hydrocarbons. Based on experimental results, an empirical relationship between the response (reaction rate) and independent variables (temperature and the partial pressures of $\mathrm{H}_{2}$ and $\mathrm{CO}$ ) was attained and expressed by the second-order polynomial equation. The investigating of these operational variables on reaction rate of FTS showed that the reaction conditions extremely influenced the rate of $\mathrm{CO}$ consumption. Effect of experimental parameters on the rate of CO consumption in FTS was established by the response surface and contour plots of the model-predicted responses. Temperature and partial pressure of CO played a significant role as well. The results showed that the reaction rate was increased with increasing the temperature and pressure. Analysis of variance showed a high coefficient of determination value $\left(\mathrm{R}^{2}=0.9902\right)$, thus ensuring a satisfactory adjustment of the second-order regression model with the experimental data. The response surface model was statistically validated by ANOVA.

Acknowledgments. The publication cost of this paper was supported by the Korean Chemical Society.

\section{REFERENCES}

1. Mansouri, M.; Atashi, H.; Mirzaei, A. A.; Jangi, R. Int. J. Ind. Chem. 2013, 4, 1.

2. Tihay, F.; Roger, A. C.; Kiennemann, A.; Pourroy, G. Catal. Today 2000, 58, 263.

3. Barrault, J.; Forquy, C.; Perrichon, V. Appl. Catal. A: General. 1983, 5, 119.

4. Barrault, J.; Forguy, C.; Menezo, J.; Maurel, R. Reac. Kinet. Catal. Lett. 1981, 17, 373.

5. Mirzaei, A. A.; Faizi, M.; Habibpour, R. Appl. Catal. A: General 2006, 306, 98.

6. Jafarzadeh, N. K.; Sharifnia, S.; Hosseini, S. N.; Rahimpour, F. Korean J. Chem. Eng. 2011, 28, 531.

7. Malleswara, T. V.; Vico-Ruiz, E.; Banares, M. A.; Deo, G. 
J. Catal. 2007, 258, 324.

8. Elavarasan, P.; Kondamudi, K.; Upadhyayule, S. Chem. Eng. J. 2009, 155, 355.

9. Mafi Gholami, R.; Mousavi, S. M.; Borghei, S. M. J. Ind. Eng. Chem. 2012, 18, 218.

10. Calemma, V. Catal. Today 2005, 106, 282.

11. Farias, F. E. M.; Sales, F. G.; Fernandes, F. A. N. J. Nat. Gas Chem. 2008, 17, 175

12. Shahrezaei, F.; Mansouri, Y.; Zinatizadeh, A. A. L.; Akhbari, A. Powder Technol. 2012, 221, 203.

13. Shafeeyan, M. S.; Daud, W. M. A. W.; Houshmand, A.; Arami-Niya, A. Fuel 2012, 94, 465.

14. Monyanon, S.; Luengnaruemitchai, A.; Pongstabodee, S. Fuel Process. Technol. 2012, 96, 160.

15. Hariharan, N. M.; Sivashanmugam, M.; Kasthurrirengan, S. J. Hydrodynamics 2013, 25, 72.

16. García-Vargas, J. M.; Valverde, J. L.; de Lucas-Consuegra, A.; Gómez-Monedero, B.; Dorado, F.; Sánchez, P. Int. J. Hydrogen Energy 2013, 38, 4524.

17. Mansouri, M.; Atashi, H.; Farshchi Tabrizi F.; Mirzaei, A. A.; mansouri, G. J. Ind. Eng. Chem. 2013, 19, 1177.

18. Montgomery, D. C. Design and Analysis of Experiments, 5th ed.; John Wiley \& Sons: New York, 2001.

19. Myers, R. H.; Montgomery, D. C. Response Surface Methodology: Process and Product Optimization Using Designed Experiments, 2nd ed.; John Wiley \& Sons: New York, 2002.

20. Gribval-Constant, A.; Khodakov, A. Y.; Bechara, R.; Zholobenko, V. L. Stud. Surf. Sci. Catal. 2002, 144, 609.

21. Atashi, H.; Siami, F.; Mirzaei, A. A.; Sarkari, M. J. Ind. Eng. Chem. 2010, 16, 952.

22. Mirzaei, A. A.; Shirzadi, B.; Atashi, H.; Mansouri, M. J. Ind. Eng. Chem. 2012, 18, 1515.

23. Fazlollahi, F.; Sarkari, M.; Zare, A.; Mirzaei, A. A.; Atashi, H. J. Ind. Eng. Chem. 2012, 18, 1223.

24. Yates, I. C.; Satterfield, C. N. Energy Fuels 1991, 5, 168.

25. van Steen, E.; Schulz, H. Appl. Catal. A: General 1999, 186, 309.

26. Ngwenya, T.; Glasser, D.; Hildebrandt, D.; Coville, N.; Mukoma, P. Ind. Eng. Chem. Res. 2005, 44, 5987.

27. Rautavuoma, A.; van der Baan, H. Appl. Catal. 1981, 1, 247.

28. Malek Abbaslou, R. M.; Slotan Mohammadzadeh, J. S.; Dalai, A. K. Fuel Process. Tech. 2009, 90, 849.

29. Liu, Y.; et al. J. Mol. Catal. A: Chem. 2007, 272, 182. 